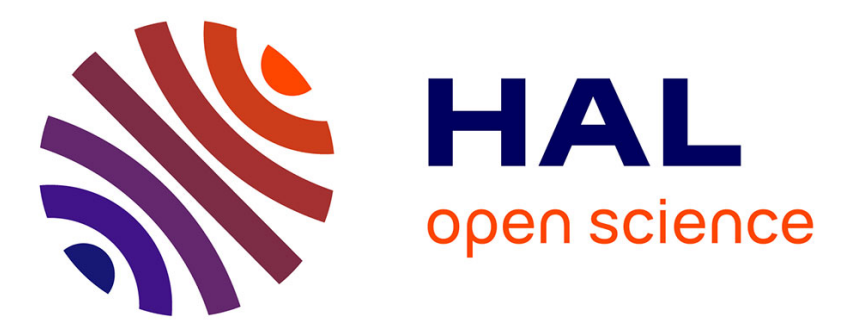

\title{
L'évolution des problématiques et des méthodologies dans l'analyse des différenciations sociales de carrières scolaires
}

Marie Duru-Bellat

\section{To cite this version:}

Marie Duru-Bellat. L'évolution des problématiques et des méthodologies dans l'analyse des différenciations sociales de carrières scolaires. Carrefours de l'éducation, 1998, 5, pp.134-145. hal-03208091

\section{HAL Id: hal-03208091 \\ https://hal.science/hal-03208091}

Submitted on 3 Jun 2021

HAL is a multi-disciplinary open access archive for the deposit and dissemination of scientific research documents, whether they are published or not. The documents may come from teaching and research institutions in France or abroad, or from public or private research centers.
L'archive ouverte pluridisciplinaire HAL, est destinée au dépôt et à la diffusion de documents scientifiques de niveau recherche, publiés ou non, émanant des établissements d'enseignement et de recherche français ou étrangers, des laboratoires publics ou privés.

\section{(이) $\$$}

Distributed under a Creative Commons Attribution - NonCommercial - NoDerivatives| 4.0 


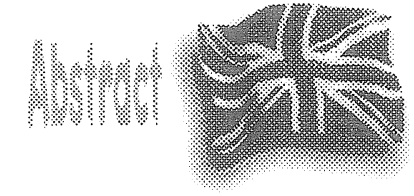

1?:? In problematics and methodologies applied to the analysis of socia differentiations of pupils'development have evolved significantly over the last thirty years. This text offers a analysis of them, distinguishing four periods 1. Before 1975, when "macro» approaches dominated, promoting school as an all-powerful institution. 2. The years 1975-1985 when the focus moved to the processes (essentially educational) through which inequalities wer created, even if the problematic of socialisation remained very significant, with a certain determinism thrown in. 3 . The years 1985 1990 when the logic of strategic action cam to the fore, leading to joint consideration of the actors and the context, especially the constraints of the situation; in particular, "teacher effects and "school effects" were "discovered", as well as the variety of family strategies. 4 . From the nineties onwards, and in parallel with the development of work on the influence of the educational context (assessment of local or nation policies, for example) ways of considering inequalities became diversified: the theme of exclusionintegration has become more and more vital, in the same way as interest in the "subjective face of inequalities or the relationships between inequelities at school and inequalities in life. This evolution leads to new theoretical questions, but also confirms an articulation to be developed between research on schooling and the political debates on the question.

The evolution of problematics and methodologies in the analysis of social differentiation of pupils' developmen
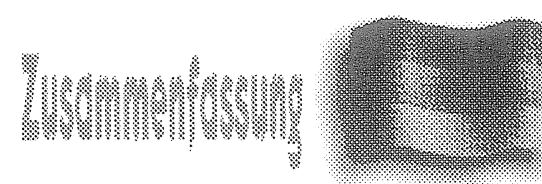

Die bei der Analyse der sozialen Differenzienung von Schulkarrieren angewandten Problematisierungs- und Methodologieansätze haben sich in den letzen dreißig Jahren empfindlich weiterentwickelt. Dieser Entwicklung geht die vorliegende Untersuchung nach und unterscheidet dabei vier Perioden: 1. Der Zeitraum vor 1975, in dem die Makroansätze dominieren welche die Schule zur allmächtigen Institution aufwerten. 2. Der Zeitraum von 1975 bis 1985, in dem sich der Blickpunkt mit einem gewissen Hang zum Determinismus auf die (vor allem schulischen) Prozesse verlagert, durch welche Ungleichheit entsteht, in dem jedoch die Problematik der Sozialisierung sehr prägnant bleibt. 3. Der Zeitraum von 1985-1990, in den die Logik strategischen Handelns bestätigt wird, welche dazu führt, daß man sich zugleich für die Handelnden und den Kontext interessiert, besonders auch fü die situationsabhängigen Zwänge; ganz besonders „ent deckt” man nun die "Lehrereffekte" und di "Institutionseffekte", aber auch die Reichhaltigkeit de Familienstrategien. 4. Ab den 90er Jahren diversifprallel zur Entwichlung der Arbeiten uber den Einfluß des Einschulungskontextes (zum Beispiel die Bewertung nationaler und kommunale politischer Maßsnahmen) - die Denkansätze hin sichtlich von Ungleichheit: Das Thema ExklusionIntegration tritt immer mehr in den Vordergrund, ebenso das Interesse an der „subjektiven Seite” der Ungleichheit oder auch die Beziehungen zwischen Ungleichheit in der Schule und Ungleichheit im Leben. Diese Entwicklung führt zu neuen theoretischen Fragestellungen, zeugt jedoch auch von eine Arbeitsteilung zwischen Forschungen uber die Schule und den diese betreffenden politischen Debatten.

Die Entwicklung von Problematisierungs- und Methodologieansäzzen bei der Analyse der sozialen Differenzierung von Schulkarrieren

\section{L'évolution des problématiques et des méthodologies dons l'analyse des différenciations sociales de corrières scoloires}

\author{
$\triangle$ Marie Duru-Bellat \\ université de Bourgogne \\ et IREDU-CNRS.
}

tement aux acteurs et au contexte, notamment aux contraintes de la situation; en particulier, on "découvre " alors les "effets maîire et les "effets établissement», mais es problématiques et aussi la variété des stratégies familes méthodologies liales. 4. À partir des anmées quatremises en oeuvre dans vingt-dix, et parallèlement au dével'analyse des différen- loppement des travaux sur l'influence ciations sociales de du contexte de scolarisation (évalluacarrières scolaires ont tion des politiques municipales ou sensiblement évolué nationales, par exemple), les durant les trente dernières années. Ce texte en propose une analyse, en distinguant quatre périodes: 1. Avant 1975, où dominent les approches «macro» valorisant une école institution toute puissante. 2. Les années 1975-1985, où le regard se déplace sur les processus (essentiellement scolaires) par lesquels se fabriquent les inégalités, même si la problématique de la socialisation reste très prégnante, avec à la clef un certain déterminisme. 3. Les années 1985-1990, où la logique de l'action stratégique s'affirme, amenant à s'intéresser conjoinmanières de penser les inégalités se diversifient: le thème de l'exclusionintégration devient de plus en plus prégnant, de même que l'intérêt pour la «face subjective » des inégalités, ou encore les relations entre inégalités à l'école et inégalités dans la vie. Cette évolution débouche sur des interrogations théoriques nouvelles, mais elle atteste également d'une articulation à travailler, entre les recherches sur l'école et les débats politiques qui la concerment. 
es problématiques et les méthodologies mises en ceuvre dans l'analyse des différenciations sociales de carrières scolaires ont sensiblement évolué durant les trente demières années. La lecture de cette évolution, proposée dans ce texte, se fait nécessairement d'un certain point de vue, et ce sera en l'occurrence celui d'une chercheuse de terrain qui a toujours cherché à lier problématiques et méthodologies... En effet, « la véritable portée d'un cadre conceptuel ne se réalise qu'à travers des choix méthodologiques» (Dubet, 994), sachant que ces choix conceptuels et méthodologiques incorporent également, nous y reviendrons, des considérations éthiques. Dans cette lecture, seront dégagées, de manière forcément arbitraire, quatre périodes.

\section{Jusqu"au milleu des anmées soixante-dix}

La première va jusqu'au milieu des années soixante-dix. Rappelons tout d'abord que jusqu'au début des années soixante, l'analyse de la variabilité de la réussite scolaire est dominée en France par des approches psychologiques ou psychopédagogiques, de ce qui ne s'appelle pas encore l'échec scolaire, comme l'ont montré les analyses de V. Isambert-Jamati (1985) sur l'émergence de cette notion. Il est clair que cette émergence est liée au développement de la scolarisation elle-même, et de la confrontation inédite, dans des structures progressivement unifiées, de population qui évoluaient jusqu'alors dans des contextes complètement disjoints.

Mais on peut aussi considérer que la construction de l'échec/la réussite scolaire comme problème sociologique a participé de la constitution même de la sociologie de l'éducation. C'est assez clair en Grande-Bretagne, où les sociologues se sont, à partir des années cinquante, positionnés contre les psychologues qui défendaient la thèse de l'héréditabilité de l'intelligence (depuis les travaux de Burt au début du siècle). Ils l'ont fait en contestant, de manière aussi péremptoire, l'hérédité de l'intelligence, en affirmant l'existence de "déterminants sociaux de l'éducabilité » (Floud et Halsey, 1958). Ils délimitaient ainsi leur domaine réservé, sur la base de ce qui constitue un postulat, puisque la question du caractère inné ou acquis de l'intelligence reste relativement indémontrable, vu le caractère d'emblée social du développement cognitif. Ce positionnement «anti-psychologues» est très révélateur de la posture, voire de l'éthique disciplinaires des sociologues; on le retrouve en France, à partir des années soixante. C'est ainsi qu'en 1964 (dans Les Héritiers), Bourdieu et Passeron posaient qu'en matière d'héréditabilité de l'intelligence, il fallait mieux «douter trop que trop peu»... Il y a là, à mes yeux, un principe moral de base commun à la plupart des sociologues.

Dans cette perspective, ce n'est pas un hasard si les sociologues français se focalisent alors sur la fonction globale du système. C'était sans doute la manière la plus «sociologiquement correcte » d'aborder la question des inégalités sociales à l'école. C'était en outre une lecture «politiquement correcte», pour la plupart des sociologues des années soixante, soixante-dix, dans un contexte idéologique très marqué par le marxisme.

Plusieurs remarques peuvent être faites, sur cette orientation privilégiée:

Les sociologues français de l'éducation ont eu tendance (dans leur majorité, à quelques exceptions dont Boudon) à considérer a priori l'école comme l'instrument unique du processus de la distribution sociale. Comme le montrent les analyses de Cuin (1995), ce «scolacentrisme» se serait davantage fondé sur des a priori théoriques et normatifs (faut-il invoquer la place dévolue à l'institution scolaire dans la société française, ou plutôt un ethnocentrisme d'intellectuels?), que sur des considérations empiriques; en effet, ni Baudelot et Establet, ni Bourdieu et Passeron n'ont analysé précisément les relations entre titres scolaires et positions sociales. Ce «scolacentrisme» aurait amené à focaliser les débats sur la démocratisation du système scolaire, puisque celui-ci était censé tenir une place centrale dans l'accès aux positions sociales. Ce «scolacentrisme» aurait aussi freiné le développement d'une réelle sociologie de la mobilité sociale. Nous y reviendrons, le développement récent d'analyses empiriques précises sur les relations formation-emplo amène par ricochet à critiquer cette approche de l'école privilégiée dans les années soixante-dix.

Seconde remarque, on comprend aisément, dans ce contexte intellectuel et idéologique des années soixante-dix, le très faible développement d'une sociologie des inégalités entre les sexes à l'école, en France. Dans un système obnubilé par la reproduction, à l'école et par l'école, des rapports de classes, le sexe a du mal à être pris au sérieux, sur le plan sociologique. Il est pourtant évident, d'un point de vue intellectuel, qu'une théorie satisfaisante du fonctionnement de l'école devrait pouvoir rendre compte tout autant des différences de réussite entre les sexes (ou les groupes ethniques) qu'entre les classes.

Troisième remarque, les méthodes privilégiées à l'époque pour vérifier (plus que véritablement démontrer) cette fonction reproductrice de l'école, sont de type enquêtes statistiques, relativement descriptives (qui fréquente tel réseau, par exemple). On peut se demander si cette prééminence des approches macrosociologiques, nourries enquêtes statistiques menées à un niveau central, n'a pas été d'autant plus marquée en France qu'il n'existait pas de lien entre la sociologie de l'éducation et la formation des maîtres (comme en Grande-Bretagne, système par ailleurs moins centralisé, où les approches «microsociologiques» se sont développées plus précocement, sans doute non sans rapport avec la demande des futurs acteurs du système)

Dernière remarque, dans le cadre de cette sociologie critique, l'école n'en reste pas moins perçue comme une institution, institution qui est véritablement toute puissante. La reproduction, c'est en effet «l'ajustement spontané des cultures scolaires aux inégalités sociales par le biais des habitus, des aspirations et des codes 
linguistiques» (Dubet, 1994). Et ce même si l'on trouve chez Baudelot et Establet, comme chez Bourdieu et Passeron, certains questionnements sur les programmes ou les interactions en classe.

\section{Les érolutions de la fin des amiées soixante=dix}

Venons-en à présent aux évolutions qui se sont amorcées dans la seconde moitié des années soixante-dix. Cette période est celle de la généralisation de la scolarisation dans le premier cycle du second degré, ce qui fait évoluer sensiblemen les modalités de la sélection sociale face à l'école, puisque celle-ci se réalise à présent au sein même du système scolaire. Les sociologues sont alors conduits à délaisser quelque peu les grandes enquêtes statistiques (d'autant plus qu'un certain nombre d'organismes ont été mis en place, qui semblent pouvoir remplir cette fonction), pour se centrer sur les processus fins par lesquels se fabriquent les inégalités de réussiteléchec. À nouveau, plusieurs remarques peuvent être faites.

Cette évolution prend place dans un contexte intellectuel où la critique du fonctionnalisme se fait de plus en plus pressante (concernant la macrosociologie de l'éducation des années soixante-dix, la dérive aurait été de considérer que tout qui se passe à l'école s'explique par sa fonction). Cette critique concerne tout particulièrement le postulat selon lequel «le système et sa cohérence déterminent l'action de chacun» (Dubet, 1994); elle débouche sur une montée des théories de laction, reconnaissant par principe aux acteurs des capacités d'initiative, et donc la possibilité de prendre quelque distance par rapport au système.

Mais de fait, la tendance à expliquer l'action par la socialisation (par les attentes, par l'adhésion à certains codes...) semble être restée très vivace, dans la sociologie de l'éducation française, au moins jusque dans ces demières années (nous y reviendrons). Et c'est la socialisation qui prend place dans le cadre scolaire qui a été privilégiée dans les recherches.

Ceci reflète sans doute une méfiance toute française, de nature autant éthique qu'intellectuelle, à l'égard des approches culturalistes, centrées sur la socialisation familiale, percues comme susceptibles de dérives du type «c'est la faute des familles ». La crainte de développer un modèle du déficit, politiquement incorrect chez la plupart des sociologues, les a conduits à se centrer sur cette éducation qui est proposée dans le cadre scolaire. On met alors en exergue combien ses contenus et ses valeurs s'enracinent dans la culture des groupes dominants. L'inégalité face à l'école n'est plus analysée alors comme la résultante du milieu social de l'élève, mais bien comme une production du système éducatif lui-même, qui construit une conception du savoir et de l'intelligence qui discrimine certains enfants.

Cette orientation a sans doute contribué à détourner lés sociologues de la recherche d'éventuelles inégalités spécifiquement cognitives, produites par l'environnement familial (à l'instar de psychologues comme Lautrey); la question de savoir si, au-delà des artefacts dans la mesure de l'intelligence, certains milieux sociaux ne sont pas à même d'inférioriser réellement certaines enfants, reste de fait taboue, même si elle est, me semble-t-il, incontournable si l'on se rallie, comme la plupart des sociologues, à une conception environnementaliste de l'intelligence.

Cela dit, cette centration sur la socialisation scolaire reflète également l'influence sur la sociologie française, de la sociologie des curricula en plein développemen en Grande-Bretagne; en attestent par exemple les travaux de Tanguy (1983) sur la conception des programmes à l'intention des «futurs ouvriers». Ce qui es caractéristique des travaux français (par contraste avec les pays anglo-saxons) c'est précisément le souci d'articuler les modalités de la socialisation scolaire et les inégalités sociales observées au niveau macro. Mais à nouveau, cette spécificité reflète autant qu'une «obsession française» pour les inégalités, de nature éthique la prégnance durable du modèle déterministe.

\section{A partir des ammées 1985}

Mais, notamment à partir des années 1985, la sociologie de l'éducation ne pouvait échapper à la montée des modèles valorisants, à côté de la logique de l'intégration/socialisation, la logique de l'action stratégique, amenant sur le devant de la scène non seulement l'acteur-stratège mais aussi le contexte de l'action et les contraintes de la situation. Elle ne pouvait y échapper non pas seulement parce que cela tendait à devenir le paradigme dominant, mais aussi parce que l'évolution même du système scolaire, connue par des travaux de plus en plus nombreux, invitait à cette évolution. En effet, parallèlement à une certaine massification du secondaire, de nouvelles différenciations se sont subtilement introduites, dans un système qui apparaît de moins en moins comme un système mais comme un espace (pour ne pas dire un marché), où les différentes filières, les diverses disciplines, les établissements sont de fait dotés de valeur différente.

À cet égard, les premiers travaux parus autour de 1985, faisant état d'« effets établissements » et d'« effets maîtres » (Duru-Bellat et Mingat, 1988; Mingat, 1984) ont joué un rôle important dans l'évolution du contexte scientifique. Ils démontrent que l'école ne peut plus être considérée comme un système monolithique fonctionnant partout de manière uniforme (avec toujours sous-jacent un fort déterminisme), ce qui conduit à admettre que le système «ne se réduit plus simplement aux contraintes qui pèsent sur lui » (comme l'a analysé Cousin, 1993).

Ce passage de l'analyse du système à celle de ses unités de production exigeai la mobilisation de méthodologies nouvelles, beaucoup plus sophistiquées que celles mises en œuvre dans les enquêtes statistiques descriptives des années soixantedix. Seuls des modèles multivariés permettent en effet de faire la part, dans les différences de résultats (au sens large) entre établissements, entre ce qui reflète des effets de composition et ce qui révèle de véritables spécificités locales (ce qu'on désigne précisément par le terme d'effet établissement). À cet égard, la faiblesse de 
la formation méthodologique et statistique des sociologues et des spécialistes de sciences de l'éducation en France, jointe bien sûr à la prégnance d'un contexte privilégiant la toute puissance du système, n'est pas étrangère au caractère tardif de l'émergence de cette problématique de l'effet établissement dans notre pays.

Cela dit, les approches dites qualitatives de ces contextes locaux se sont largement développées sur la période. Sont ainsi mises en exergue les multiples facettes de l'action des acteurs, qui construisent leur parcours scolaire (ou celui de leur enfant) à partir de leur position sociale et à travers des stratégies spécifiques (Berthelot initie ce type de perspective au début des années quatre-vingt). On assiste également à un fort développement des travaux sur les familles et leurs relations avec l'école (notamment ceux menés par Henriot van Zanten, 1988); ou encore, à la suite à l'assouplissement de la carte scolaire en 1984, des travaux sur les stratégies de choix d'établissement, au sein du public et entre le public et privé (notamment Langouât et Léger, 1991). Se développent aussi des analyses plus fines du pilotage par les familles des carrières scolaires de leur enfant (choix d'option et d'orientation, au sein d'un système de plus en plus diversifié), sur la base de méthodes plus sophistiquées (cf. par exemple Duru-Bellat et al., 1993), permettant de distinguer réussite et orientation, et d'analyser la genèse des inégalités sociales sur la base de concepts aujourd'hui banalisés, tels que l'autosélection.

Ce qu'il faut souligner, c'est que ces travaux sur les effets du contexte institutionnel, gardent en ligne de mire le souci d'expliquer la genèse des inégalités macrosociales, même sils le font sur la base de problématiques et de méthodologies différentes.

\section{Remargues sur le contexte de la demière décennie}

Quelques remarques, pour finir, sur le contexte de la dernière décennie.

Tout se passe comme si les inégalités sociales face à l'école étaient à présent considérées comme acquises (et bien connues, grâce aux données de la DEP notamment), le travail du sociologue consistant essentiellement à explorer la «boite noire». Au moins dans le champ de la sociologie (et alors que les didactiques se sont beaucoup développées), les recherches restent encore peu nombreuses concernant l'évolution des pratiques pédagogiques (Demailly, 1991), et leurs effets auprès des élèves (cf. par exemple Tupin, 1996, ou Suchaut, 1996); ce type de recherche rencontre en France des problèmes d'accès au terrain et de méthode importants.

Les travaux sur l'influence du contexte de scolarisation ont par contre continue à développer: travaux sur la variété des établissements, travaux sur les effets des politiques publiques (ZEP par exemple, cf. Meuret, 1994), ou des politiques régionales ou municipales (Henriot van Zanten, 1990; Charlot, 1994), ou encore les modes d'organisation du système, tels que le groupement des élèves par niveau (Duru-Bellat et Mingat, 1997). Mais pour articuler l'analyse de ces différents «niveaux de contexte» (classe et établissement notamment), il conviendrait de recourir à des méthodes de plus en plus sophistiquées (modèles multiniveaux), ce qui pose des problèmes spécifiques dans le contexte français (où à nouveau la recherche apparaît relativement « en retard » par rapport aux pays voisins).

Néanmoins, il semble aujourd'hui admis que la prise en compte du contexte n'a pas un caractère accessoire, dans l'analyse des inégalités sociales; c'est en effet dans un contexte qui constitue un ensemble de contraintes que l'acteur prend ses décisions et apprend. Pour évaluer les effets de ces caractéristiques du contexte sur les inégalités sociales à l'école (qu'il s'agisse d'acquis, de carrière ou d'expérience scolaire), les évaluer mais aussi les comprendre, des articulations entre approches quantitatives et qualitatives sont sans doute à terme incontournables.

Trois autres particularités des recherches produites dans ce contexte des années quatre-vingt-dix méritent être soulignées

Tout d'abord, l'évolution du contexte économique (montée du chômage et de l'exclusion) amène à délaisser quelque peu le thème de la démocratisation de l'école elle-même, au profit des rapports entre l'école et l'environnement économique et social. Avec par conséquent un développement d'interrogations sur le rôle de l'école dans l'intégration ou l'exclusion des différents groupes sociaux (van Zanten, 1996)

Par ailleurs, apparaissent des approches qui osent s'intéresser à la «face subjective» de la vie scolaire (alors que cela a longtemps constitué un interdit pour les sociologues). Ceci passe par l'élaboration de nouveaux concepts, tels que le rapport au savoir (Charlot et al., 1992; Rochex, 1995), ou dans une perspective différente celui d'expérience scolaire (Dubet, 1994). L'évolution même du système éducatif, à nouveau, n'est sans doute pas étrangère à cette évolution (ouverture à de nouveaux publics, montée des préoccupations stratégiques des élèves) Mais elle relève aussi d'un approfondissement de la réflexion des sociologues sur les fonctions de l'école, et aussi d'une conception «post-moderne » d'un sujet qui ne peut plus se définir uniquement par des rôles sociaux et a à construire sa subjectivité.

Ce type d'approche est tout à fait heuristique pour penser, vieille obsession des sociologues, l'articulation micro/macro, puisque la subjectivité des acteurs sociaux est rapportée aux mécanismes objectifs qui la produisent. Comme le soulignent Dubet et Martucelli (1996), «si chacun construit lui-même son expérience sociale, il n'empêche qu'il ne la bâtit qu'avec des matériaux «objectifs» et qui ne lui appartiennent pas» (relations entre cultures familiales, culture scolaire, utilités sociales objectives attachées aux études, contenus scolaires et méthodes pédagogiques). En outre, ce type d'approche met en exergue une dimension nouvelle des inégalités à l'école, à savoir une hiérarchie des expériences des élèves, où les «dominés» vont avoir des difficultés spécifiques à résoudre les tensions qui caractérisent leur expérience scolaire (Dubet et Martucelli, 1996). Avec du même coup, un développement des travaux autour de la déviance et de la violence à l'école.

Au total, avec cette découverte de la subjectivité des élèves, se renforce un glis- 
sement significatif de la perspective sociologique sur l'école, qui n'est plus considérée seulement comme un cadre (où s'expriment des déterminismes sociaux extérieurs), mais bien comme le lieu d'une activité spécifique des acteurs, notamment des élèves (construction de savoirs, construction d'une expérience).

Enfin, autre aspect notable de ce contexte des années quatre-vingt-dix, le développement de travaux portant sur d'autres «variables» que l'origine sociale, le sexe et la nationalité notamment. Ils amènent à déplacer l'attention vers d'autres facettes des inégalités sociales à l'école et sur la variété des «ingrédients » nécessaires pour y réussir. Mais on peut noter qu'ils restent souvent conduits dans un perspective très "années soixante, soixante-dix», où les enfants sont considérés comme des victimes de l'organisation du système (par exemple, les garçons « victimes de l'enfance des chefs », comme l'expriment de manière suggestive Baudelo et Establet). La prise en compte de la variable sexe permet pourtant de pointer des «anomalies » heuristiques. Ainsi, les filles réussissent relativement bien à l'école malgré une socialisation scolaire à maints égards défavorable (interactions pédagogiques moins stimulantes, confrontation à des contenus de formation souvent marqués par le sexisme...); et ensuite, sur le marché du travail, cette relative réussite scolaire apparaît guère payante, de même qu'il ne suffit pas à un enfant issu de l'immigration être diplômé pour s'insérer sans problème.

Si les interrogations théoriques que pourrait susciter cette anomalie restent peu développées, c'est sans doute parce qu'elles remettraient profondément en cause la façon de penser l'école. Car ce constat comme quoi le «traitement pédagogique » des filles en classe est peu lié à leur réussite, elle-même peu liée à leur insertion ultérieure, est effectivement perturbant (cf. Moore, 1996): il montre qu'il n'existe pas de relation linéaire, mécanique, entre la socialisation scolaire, la réussite et les opportunités sociales, comme le posait le schéma classique de la reproduction. En particulier, les effets de l'éducation ne sont-ils pas en fait relativement faibles par rapport à l'influence de ce qui prend place hors de l'école (les filles anticipant par exemple les exigences particulièrement élevées du marché du travail à leur encontre)? Mais cela conduirait à admettre que l'éducation n'est pas forcément cet instrument de changement social dans lequel on a mis tant d'espoir...

Mais cette question est plus que jamais d'actualité. Des travaux récents ont remis sur le devant de la scène la question de l'articulation entre inégalités à l'école et inégalités dans la vie (en France, cf. les travaux d'Euriat et Thélot, Merle, Goux et Maurin). En France comme au niveau européen, on assiste à la fois à un fort développement de l'éducation et au maintien d'inégalités sociales importantes; les seuls pays où une certaine démocratisation des carrières scolaires a été observée (PaysBas ou Suède) sont ceux où l'on a assisté à une réduction des inégalités sociales de niveau de vie et de sécurité économique. Une question est alors de savoir quel peut être le modèle théorique le plus pertinent, sachant qu'ün modèle théorique est censé permettre de comprendre la réalité. Actuellement, on redécouvre beaucoup à l'étranger la portée des analyses de Boudon (cf. Goldthorpe, 1996). En effet, le modèle d'un acteur qui évalue, en fonction de sa position sociale, les risques et les enjeux sociaux des différentes alternatives scolaires peut apparaître intéressant pour comprendre la translation des inégalités; c'est ce que proposait Boudon, mais c'est aussi ce que suggéraient Bourdieu et Passeron quand ils mettaient en avant la fonction de distinction des titres scolaires.

Dans cette perspective, c'est l'appropriation stratégique de l'école par des groupes sociaux inégaux qui constitue un des freins les plus puissants à une réelle démocratisation de l'école. Avec pour conséquence qu'une réelle démocratisation ne peut venir que de deux sources: 1 . Soit d'une réduction des écarts entre groupes sociaux (qui affectent à la fois leurs ressources et ces différents paramètres de la prise de décision que sont le risque ou l'enjeu attaché à telle ou telle alternative éducative); 2. Soit d'un relâchement des liens entre formation et emploi qui produisent cette «diffusion régressive des enjeux» dont parle Berthelot (pour bien s'insérer socialement, il faut se classer parmi les premiers, pour ce faire, il faut constituer telle filière en filière d'excellence, et dévaloriser corrélativement toutes les autres etc.)

Prost ne disait pas autre chose dans les années quatre-vingt (Prost, 1985): dans la mesure où la demande des familles est sociale - demande de classement et non demande intrinsèque de formation -, tout le monde ne peut, par définition, réussir. Pour casser cette nécessité de l'échec scolaire, il faut sans doute remettre en cause la force de la relation entre formation et emploi que l'on avait eu tendance à considérer, en France, comme la condition d'une société démocratique. Mais même s'il est clair qu' « une moindre compétition pour le statut social dans la population modifierait le climat des classes », la question des critères alternatifs, pour l'allocation dans les emplois, reste ouverte... Rappelons que Prost suggérait également, proposition sans doute moins discutable, de redonner sa place à une véritable formation de base, où l'on valoriserait «les justifications scolaires de préférence aux justifications sociales externes».

Ces débats, qui posent in fine la question de la justice dans le système scolaire, sont amenés à prendre une importance politique croissante, et si la sociologie de l'éducation (et les Sciences de l'Éducation) ne veulent pas se voir cantonnées à des activités d'expertise sociale, elles devront immanquablement s'y atteler, sur une base à la fois conceptuelle, empirique, mais aussi, inévitablement, éthique.

NOTE

Ce texte reprend sous une forme remaniée une intervention faite le 12 décembre 1997 dans le cadre du colloque «À Paris V, 30 ans de sciences de l'Éducation».

\section{BIBLIOGRAPHIE}

Baudelot (C.), Establet (R.), 1971, L'école capitaliste en France, Paris, Maspero. Baudelot (C.), Establet (R.), 1992, Allez les filles, Paris, Seuil.

Berthelot (J.-M.), 1983, Le piège scolaire, Paris, PUF. 
Berthelot (I-M) 1993 École, orientation, société Paris, PUF

Boudon (R.), 1973, L'inégalité des chances. La mobilité sociale dans les sociétés industrielles, Paris, Colin. Bourdieu (P.), Passeron (J.-C.), Chamborédon (J.-C)., 1964, Les Héritiers. Les étudiants et la culture, Paris, Ed. de Minuit.

Bourdieu (P.), 1966, «L'école conservatrice. Les inégalités devant l'école et devant la culture», Revue Française de Sociologie, VII, 325-347.

Bourdieu (P.), Passeron (J.-C.), 1970, La reproduction. Éléments pour une théorie du système d'enseignement, Paris, Ed. de Minuit.

Bourdieu (P.), 1979, La distinction. Critique sociale du jugement, Paris, Ed. de Minuit.

Bressoux (P.), 1994, "Les recherches sur les effets-école et les effets-maîres \#, Revue Française de Pédagogie, n $108,91-137$

Broccholichi (S.), 1995, "Orientations et ségrégations nouvelles dans l'enseignement secondaire " Sociétés contemporaines, $n^{\circ} 21,1527$.

Caillé (J.-P.), Vallet (L.-A.), 1996, « Les élèves étrangers ou issus de limmigration dans l'école française", Les Dossiers d'Éducation et Formations, $n^{\circ} 67$.

Charlot (B.), Bautier (E.), Rochex (J.-Y.), 1992, École et savoirs dans les banlieues... et ailleurs, Paris, Colin

Charlot (B.) (éd.), 1994, L'école et le territoire, Paris, Colin

Cousin (O.), 1993, «L'effet établissement. Construction d'une problématique », Revue française de sociologie, 34, 3, 395-419

Cuin (Ch.), 1993, Les sociologues et la mobilité sociale, Paris, PUF.

Demailly (L.), 1991, Le collège. Crises, mythe et métiers, Lille, PUL

Dubet (F.), 1994, Sociologie de l'expérience, Paris, Seuil.

Dubet (F.), Martucelli (D.), 1996, À l'école, Paris, Seuil.

Duru-Bellat (M.), 1990, L'école des filles. Quelle formation, pour quels rôles sociaux? Paris, L'Harmattan. Duru-Bellat (M.), Henriot-van-Zanten, 1992, Sociologie de l'école, Paris, Colin.

Dura Bella (M), Mingat (A), 1988, ule deroulene de la Duru-Bellat (M.), Mingat (A.), 1988, «Le déroulement de
des différences », Revue Française de Sociologie, 29, 649-666

Duru-Bellat (M.), Jarousse (J.-P.), Mingat (A.), 1993, «Les scolarités de la maternelle au lycée», Revue Française de Sociologie, 34, n-1, 43-60.

Duru-Bellat (M.) Mingat (A.), 1997, «La constitution de classes de niveau par les collèges et ses incidences sur les progressions des élèves», Revue Française de Sociologie, n 4 (à paraître).

Duru-Bellat (M.), Merle (P.), 1997, «La démocratisation impossible? Usages sociaux de l'école et inégalités sociales des cursus scolaires ", Savoir, nº 3-4.

Euriat (M.), Thélot, 1995, «Le recrutement social de l'élite scolaire depuis 40 ans», Éducation et Formations, $n^{\circ} 41,3-21$.

Felouzis (G.), 1997 . L'efficacité des enseignants, Paris, PUF.

Floud (J.), Halsey (A. H.), 1958, «The sociology of education: A trend report and bibliography », Current Sociology, VII, 3, 165-235

Goldthorpe (J.H.), 1996, "Class analysis and the reorientation of class theory: the case of persisting differentials in educational attainment », British Journal of Sociology, vol.47, $\mathrm{n}^{\circ}$ 3, 481-505.

Goux (D.), Maurin (E.), 1997, «Destinées sociales: le rôle de l'école et du milieu d'origine», Économie et Statistique, $\mathrm{n}^{\circ}$ 306, 13-26.

Goux (D.), Maurin (E.), 1997, «Démocratisation de l'école et persistance des inégalités», Économie et Statistique, $n^{\circ} 306,27-40$

Grisay (A.), 1997 «L'évolution des acquis cognitifs et socioaffectifs des élèves au cours d'années de collège», Dossiers Éducation et Formation, $n^{\circ} 88$.

Floud (J.), Halsey (A. H.), 1958, «The sociology of education: A trend report and bibliography », Current Sociology, VIl, 3, 165-235.

Henriot-van-Zanten A., 1988, «Les familles face à l'école: rapports institutionnels et relations sociales», in Durning P. (ed), Éducation familiale, Paris, MIRE/Matrice, 185-207.
Henriot-van-Zanten (A), 1990, I'école et l'espace local, Lyon, PUL

Henriot-van-Zanten (A.), Migeot-Alvarado J., 1994, «Socialisation familiale et investissement scolaire », L'Année Sociologique, $\mathrm{n}^{\circ} 44$

Héran (F.), 1996, «École publique, école privée : qui peut choisir? », Économie et Statistique, n 293 $17-39$

Isambert-Jamati (V.), 1984, Culture technique et critique sociale à l'école élémentaire, Paris, PUF.

Isambert-Jamati (V.), 1985, «Quelques rappels de l'émergence de l'échec scolaire comme «pro-

blème social» dans les milieux pédagogiques français», in Plaisance E. (éd.).

Isambert-Jamati (V.) 1990, Les savoirs scolaires, Paris Éditions universitaires.

Lahire (B.), 1993, Culture écrite et inégalités scolaires, Lyon, PUL.

Langouët (G.), 1982, Technologie de l'éducation et démocratisation de l'enseignement, Paris, PUF

Langouët (G.), Léger (A.), 1991, Public ou privé? La Garenne-Colombes, Publidix.

Langouët (G.), Léger (A.), 1997, Le choix des familles, Paris, Fabert.

Merle (P.), 1996, «Les transformations sociodémographiques des filières de l'enseignement supérieur de 1985 à 1995 », Population, 6, 1181-1210.

Meuret (D.), 1994, «L'efficacité de la politique des zones prioritaires dans les collèges», Revue

Française de Pédagogie, n ${ }^{\circ}$ 109, 41-64.

Mingat (A.), 1984, «Les acquisitions scolaires de l'élève au CP. les origines des différences», Revue Française de Pédagogie, $\mathrm{n}^{\circ}$ 69, 49-63.

Moore (R.), 1996, «Back to the Future: the problem of change and the possibilities of advance in the sociology of education", British Journal of Sociology of Éducation, vol.17, n² 2, 145-161.

Müller (W.), Karle (W.), 1993, "Social Selection in Educational Systems in Europe », European

Sociological Review, vol. 9, May, p. 1-23.

Plaisance (E.), (éd.), 1985, «L'échec scolaire», nouveaux débats, nouvelles approches sociologiques, Paris, Ed. du CNRS.

Prost (A.), 1985, «L'échec scolaire: usage social et usage scolaire de l'orientation», in Plaisance E. (éd.).

Rochex (J.-Y.), 1995, Le sens de l'expérience scolaire: entre activité et subjectivité, Paris, PUF.

Suchot (B.), 1996, «La gestion du temps à l'école maternelle et primaire: diversité des pratiques et effets sur les acquisitions des élèves», L'Année de la Recherche en Ëducation, 123-153.

Tanguy (L.), 1983, «Les savoirs enseignés aux futurs ouvriers», Sociologie du Travail, nº 3, 336354.

Terrail (J.-P.), 1997, «La sociologie des interactions famille/école», Sociétés Contemporaines, n² 25 , 67-84.

Thrupp (M.), 1995, «The School Mix Effect: the history of an enduring problem in educational research, policy and practice », British Journal of Sociology of Éducation, 16, $\mathrm{n}^{\circ} 2,183-203$.

Tupin (F.), 1996, «Espace d'action des enseignants et démocratisation: l'exemple de l'enseignement de la narration écrite au collège », Revue Française de Pédagogie, n ${ }^{\circ} 115,77-67$.

Zanten (van) (A.), 1996, «Fabrication et effets de la ségrégation scolaire», in S. Paugam (dir.), L'exclusion, l'état des savoirs, Paris, La Découverte. 